\title{
Abdominal tuberculosis CT-scan findings in comparison to malignant ovarian tumor CT-scan findings in general, a retrospective observation
}

\author{
Elwin $^{\text {a* }}$, Lies Mardiyana ${ }^{\mathrm{b}}$, Bambang Soeprijanto $^{\mathrm{b}}$, Hari Nugroho $^{\mathrm{c}}$ \\ a elwinjunaedi@gmail.com \\ ${ }^{a}$ Resident of Radiology Department, Faculty of Medicine, Airlangga University/Dr.Soetomo General Hospital, Surabaya \\ ${ }^{b}$ Staff of Radiology Department, Faculty of Medicine, Airlangga University/Dr.Soetomo General Hospital, Surabaya \\ 'Staff of Obstetric and Gynecology Department, Faculty of Medicine, Airlangga University/Dr.Soetomo General Hospital, Surabaya
}

\begin{abstract}
Background: Abdominal TB is an infection caused by Mycobacterium TB (TB) that involves the gastrointestinal tract, peritoneum, lymph nodes, and/or solid organs. Female pelvic TB is a relatively rare type of extrapulmonary TB, and little literature has been reported to date. Because of its rarity, computed tomography (CT) findings of female pelvic tuberculosis have not been fully elucidated and are easily misdiagnosed as Advanced Ovarian Malignancy (AOM) or Pelvic Inflammatory Disease (PID) without pathological findings. This study aimed to determine abdominal TB CT-Scan findings that resembled malignant ovarian tumor. Methods: This study was a descriptive study with a retrospective design using patient medical records. The study population was abdominal tumor patients who were suspected with malignant ovarian tumor and were examined by CT-Scan. Data taken from medical records of ovarian tumor patients were patients' characteristics and CT findings of abdominal TB tumor patients that resembled malignant ovarian tumor.

Results: From 22 samples, most groups belonged to 21-40-years age group. Moss masses were bilateral (46.6\%), had solid cystic component $(63.6 \%)$, lobulated contour $(50 \%)$, and all mass presented with enhancement. Ascites $(81.9 \%)$, peritoneal thickening and enhancement (68.2\%), and omental involvement (54\%) were also found. Conclusion: CT-scan findings in abdominal tuberculosis patients which resembled malignant ovarian tumors have similarities with findings in previous literatures, such as mass, ascites, and omental involvement findings
\end{abstract}

Published by IJRP.ORG. Selection and/or peer-review under responsibility of International Journal of Research Publications (IJRP.ORG)

Keywords: abdominal TB, CT scan, malignant ovarian tumor

\section{Introduction}

According to Word Health Organization (WHO) in 2014, as many as 9.6 million people contracted tuberculosis (TB) and 1.5 million people died from TB. Indonesia ranked second as a country with the highest TB prevalence in Southeast Asia after East Timor. In 2014, TB prevalence in Indonesia 297 per 100,000 population, while the incidence was 399 cases per 100,000 population (WHO, 2014; KEMENKES, 2018). 
Abdominal TB is an infection caused by Mycobacterium TB (TB) that involves the gastrointestinal tract, peritoneum, lymph nodes, and/or solid organs (Avuja, 2019). Clinical findings of abdominal TB closely resembles an intra-abdominal mass, where patients often complain of an enlarged stomach, bowel disorders and a feeling of fullness in abdominal region. This is complicated by the fact that in patients with abdominal tuberculosis, there is also an increase in tumor marker Ca-125, which is commonly used as a marker for ovarian tumors, therefore it is often misinterpreted and misdiagnosed which results in inaccurate therapy (Anita et al., 2017; Kim et al., 2017; Patel et al., 2012).

Female pelvic TB is a relatively rare type of extrapulmonary TB, and little literature has been reported to date. Because of its rarity, computed tomography (CT) findings of female pelvic tuberculosis have not been fully elucidated and are easily misdiagnosed as Advanced Ovarian Malignancy (AOM) or Pelvic Inflammatory Disease (PID) without pathological findings. CT findings interpretation and differentiation of AOM, which could metastasize, is clinically important. Understanding female pelvic TB CT findings spectrum might help diagnosis (Sah et al., 2017). This study aimed to determine abdominal TB CT-Scan findings that resembled malignant ovarian tumor.

\section{Methods}

\subsection{Study Design}

This study was a descriptive study with a retrospective design using patient medical records that was conducted at Diagnostic Radiology Installation of Integrated Diagnostic Center of Dr. Soetomo General Hospital, Surabaya, Indonesia from January 2015 - June 2020.

\subsection{Study Population and Sample}

The study population was abdominal tumor patients who were suspected with malignant ovarian tumor and were examined by CT-Scan, with the final diagnosis being abdominal TB. The study samples were all populations that met the following inclusion criteria:

1. Patients with clinical ovarian tumors who underwent CT scan.

2. Patients who underwent laparotomy surgery and were biopsied or underwent partial or complete intraabdominal tumor removal

3. Patients who were performed histopathological examinations.

4. Patients with final diagnosis of abdominal TB.

The exclusion criteria were incomplete or not readable CT-Scan raw data

\subsection{Data collection and analysis}

Data taken from medical records of ovarian tumor patients were patients' characteristics and CT findings of abdominal TB tumor patients that resembled malignant ovarian tumor. Furthermore, data from patients who had final diagnosis of abdominal TB were tabulated. Data are presented in frequency and percentage. Data analysis was conducted by descriptive analysis.

\section{Results}

\subsection{Sample characteristics based on age}

Abdominal TB patients who underwent CT-Scan examination had the youngest age of 20 years and the oldest age of 52 years. Mean age of abdominal TB patients was $32.22 \pm 10.61$ years. Patients were divided into several age groups with a range of 20 years. Most abdominal TB patients were in 21-40-years age group, which consisted of 14 people (63.6\%), 8 people in 41-60-years age group (36.4\%), and no patients were in 1-20-years age group and in 61-80-years age group.

Table 1. Abdominal TB patients' distribution based on age groups 


\begin{tabular}{lrr}
\hline \multicolumn{1}{c}{ Age } & Frequency $(\mathrm{n})$ & Percentage $(\%)$ \\
\hline $1-20$ years & 0 & 0.0 \\
$21-40$ years & 14 & 63.6 \\
$41-60$ years & 8 & 36.4 \\
$61-80$ years & 0 & 0.0 \\
\hline Total & 22 & 100.0 \\
\hline
\end{tabular}

\subsection{Pelvic Mass Findings on Abdominal CT-Scan in Abdominal TB Patients}

Abdominal CT-scan could show the presence or absence of pelvic masses in abdominal TB patients. The results showed that pelvic masses were found in 15 people (68.2\%) and no pelvic mass was found in 7 people $(31.8 \%)$.

Table 2. Distribution based on pelvic mass presence

\begin{tabular}{lrrr}
\hline & Presence of pelvic mass & Frequency $(\mathrm{n})$ & Percentage (\%) \\
\hline Yes & 15 & 68.2 \\
No & 7 & 31.8 \\
\hline Total & 22 & 100.0 \\
\hline
\end{tabular}

From a total of 15 patients with abdominal tuberculosis who had pelvic masses, the location of pelvic masses in right adnexa were found in 3 people (20.0\%), left adnexa were in 5 people (33.3\%), and both adnexa were in 7 people (46.6\%), where a total mass of 22 masses were found.

Table 3. Distribution based on pelvic mass location

\begin{tabular}{lrr}
\hline \multicolumn{1}{c}{ Pelvic mass location } & Frequency $(\mathrm{n})$ & \multicolumn{1}{c}{ Percentage (\%) } \\
\hline Right adnexa & 3 & 20.0 \\
Left adnexa & 5 & 33.3 \\
Bilateral & 7 & 46.6 \\
\hline Total & 15 & 100.0 \\
\hline
\end{tabular}

Of the 15 patients with abdominal tuberculosis who had pelvic masses, a total of 22 masses were obtained, where none had solid component, 8 masses had cystic component (36.4\%), and 14 masses had solid cystic component $(63.6 \%)$

Table 4. Distribution based on pelvic mass components

\begin{tabular}{lrr}
\hline \multicolumn{1}{c}{ Pelvic mass component } & Frequency (n) & Percentage (\%) \\
\hline Solid & 0 & 0.0 \\
Cystic & 8 & 36.4 \\
Solid cystic & 14 & 63.6 \\
\hline Total & 22 & 100.0 \\
\hline
\end{tabular}

Of the 22 masses, 5 masses had round contours (22.7\%), 11 masses had lobulated contours (50.0\%), 2 masses had irregular contours (9.1\%), and 4 masses had ovoid contours (18.2\%).

Table 5. Distribution based on the pelvic mass contour 


\begin{tabular}{lrr}
\hline \multicolumn{1}{c}{ Pelvic mass contour } & Frequency (n) & Percentage (\%) \\
\hline Round & 5 & 22.7 \\
Lobulated & 11 & 50.0 \\
Irreguler & 2 & 9.1 \\
Ovoid & 4 & 18.2 \\
\hline Total & 22 & 100.0 \\
\hline
\end{tabular}

Of the 14 masses that had solid component, which was solid cystic components, enhancements were found in all masses.

Table 6. Distribution based on solid component enhancement of pelvic mass

\begin{tabular}{lrrr}
\hline & Solid component enhancement & Frequency (n) & Percentage (\%) \\
\hline Yes & 14 & 100 \\
No & 0 & 0 \\
\hline Total & 14 & 100 \\
\hline
\end{tabular}

\subsection{Another Abdominal CT-Scan Findings in Abdominal TB Patients}

Of the 22 patients with abdominal tuberculosis who underwent abdominal CT-scan, 18 people $(81.9 \%)$ had ascites and 4 people $(18.2 \%)$ had no ascites.

Table 7. Distribution based on the presence of ascites

\begin{tabular}{lrrr}
\hline & Prescence of ascites & Frequency $(\mathrm{n})$ & Percentage $(\%)$ \\
\hline Yes & 18 & 81.9 \\
No & 4 & 18.2 \\
\hline Total & 22 & 100.0 \\
\hline
\end{tabular}

Of the 18 patients with ascites in Abdominal TB patients who underwent abdominal CT-scan, 12 people $(66.6 \%)$ had free ascites and 6 people $(33.3 \%)$ had loculated ascites.

Table 8. Distribution based on ascites type

\begin{tabular}{lrr}
\hline \multicolumn{1}{c}{ Ascites type } & Frequency (n) & Percentage (\%) \\
\hline Free ascites & 12 & 66.6 \\
Loculated ascites & 6 & 33.3 \\
\hline Total & 18 & 100.0 \\
\hline
\end{tabular}

Of the 22 Abdominal TB patients who underwent abdominal CT-Scan, 15 people $(68 \%)$ had peritoneal thickening and enhancement and 7 people (31.8\%) did not have them.

Table 9. Distribution based on peritoneal thickening and enhancement presences

\begin{tabular}{lrr}
\hline \multicolumn{1}{c}{ Peritoneal thickening and enhancement presences } & Frequency $(\mathrm{n})$ & Percentage $(\%)$ \\
\hline Yes & 15 & 68.0 \\
No & 7 & 31.8 \\
\hline
\end{tabular}




\begin{tabular}{lrr}
\hline Peritoneal thickening and enhancement presences & Frequency $(\mathrm{n})$ & Percentage $(\%)$ \\
\hline Total & 22 & 100.0 \\
\hline
\end{tabular}

Of the 15 patients with peritoneal thickening and enhancement, 14 people (93.3\%) had smooth type, and one person $(6.7 \%)$ had irregular type.

Table 10. Distribution based on peritoneal thickening and enhancement type

\begin{tabular}{lrr}
\hline \multicolumn{1}{c}{ Peritoneal thickening and enhancement type } & Frequency $(\mathrm{n})$ & Percentage (\%) \\
\hline Smooth & 14 & 93.3 \\
Irreguler & 1 & 6.7 \\
Total & 15 & 100.0
\end{tabular}

Of the 22 patients with abdominal tuberculosis, omental involvements were presence in 12 people $(54.5 \%)$ and none in 10 people $(45.5 \%)$.

Table 11. Distribution based on omental involvement

\begin{tabular}{|c|c|c|}
\hline Omental involvement presence & Frequency $(\mathrm{n})$ & Percentage (\%) \\
\hline Yes & 12 & 54.5 \\
\hline No & 10 & 45.5 \\
\hline Total & 22 & 100.0 \\
\hline
\end{tabular}

Of the 12 abdominal TB patients with omental involvement, 7 people (58.3\%) had smudged type, 3 people $(25.0 \%)$ had cake-like type, and 2 people (16.6\%) had nodular type.

Table 12. Distribution based on omental involvement type

\begin{tabular}{lrr}
\hline \multicolumn{1}{c}{ Omental involvement type } & Frequency (n) & Percentage (\%) \\
\hline Smudged & 7 & 58.3 \\
Nodular & 2 & 16.6 \\
Cake-like & 3 & 25.0 \\
\hline Total & 12 & 100.0 \\
\hline
\end{tabular}

Of the 22 Abdominal TB Abdomen patients, 8 people (4.5\%) had lymphadenopathy, and 14 people (95.5\%) had no lymphadenopathy.

Table 13. Distribution based on lymphadenopathy presence

\begin{tabular}{|c|c|c|}
\hline Lymphadenopathy presence & Frequency $(\mathrm{n})$ & Percentage (\%) \\
\hline Yes & 8 & 4.5 \\
\hline No & 14 & 95.5 \\
\hline Total & 22 & 100.0 \\
\hline
\end{tabular}

Of the 8 patients who had lymphadenopathy, 5 people $(62.5 \%)$ had central necrosis lymphadenopathy, and 3 people $(37.5 \%)$ did not have it. 
Table 14. Distribution based on central necrosis presence

\begin{tabular}{lrrr}
\hline & Central necrosis presence & Frequency $(\mathrm{n})$ & \multicolumn{1}{r}{ Percentage (\%) } \\
\hline Yes & 5 & 62.5 \\
No & 3 & 37.5 \\
\hline Total & 8 & 100.0 \\
\hline
\end{tabular}

\section{Discussion}

In this study, the youngest patient was 20 years old and the oldest was 52 years old. Abdominal TB patients in this study were mostly belonged to 21-40-years age group. The age distribution in this study was similar to a study conducted by Sah et al, which found that 15-67-years age group was the group with the highest Abdominal TB patients. Pelvic TB affected more people with reproductive age, with limited locations in fallopian tubes, endometrium, and ovaries (Sah et al., 2017).

From 22 samples, pelvic mass was found in of 15 patients (68.2\%). Pelvic mass presence in this study was similar to a study conducted by Sah, et al, where they found that $87.5 \%$ of samples had mass in their pelvis. A study by Delgado et al also reported $85.7 \%$ of samples had adnexa lesions (Sah et al., 2017; Delgado et al., 2017).

In terms of location, most patients had bilateral mass (46.6\%), and the total mass obtained were 22 masses. This finding was similar to study conducted by Wang et al., (2019), where they reported most patients (53.33\%) had bilateral mass. Abdominal tuberculosis patients could have masses in right, left, or both adnexa. Thankam and Varma (2008) stated that ovarian TB usually occurs bilaterally, it could occur because most of TB extensions come from fallopian tubes. From previous data, more than $90 \%$ of genital tubal TB is bilateral.

In our study, mass with cystic and solid cystic components were found, where most of them had solid cystic component. This was in accordance to a literature which stated that adnexal masses findings in abdominal TB could be either cystic, or solid cystic (Kim et al., 2004)). A study from Sah et al., (2017) also reported that solid cystic was the most frequent mass component in their study. However, apart from ascites, peritoneal thickening and enhancement, and omental involvement, mass findings could also lead to an AOM and PID (Sah et al., 2017; Kim et al., 2004). However, previous literatures stated that simple ovarian cyst could be round or oval in shape without solid component, was smooth, and thin-walled, while malignant cyst appeared to be solid inside (Ross and Fortin, 2016; Valentini et al., 2012). Thus, we concluded pelvic mass components in the form of cystic or solid cystic tuberculosis is not pathognomonic, because it could also be signs of benign or malignant ovarian masses.

In this study, $22.7 \%$ of the masses were round-shaped, $50 \%$ of the masses were lobulated-shaped, $9.1 \%$ of the masses were irregular-shaped, and $18.2 \%$ of the masses were ovoid-shaped. This was different from a study by Sah et al., (2017) which found that from 28 masses of pelvic TB patients, there were 13 round contours, 15 lobulated masses, one tubular mass, and 12 ovoid masses. So far, no other studies have similar results. However, from previous studies above, there are various variations in pelvic masses contours, therefore it cannot be used as pathognomonic for abdominal tuberculosis characteristics.

All solid mass presented with enhancement in this study. This was in accordance to a study by Sah et al., (2017) which reported pelvic mass enhancement from an average of $34 \mathrm{HU}$ in pre contrast, to $48 \mathrm{HU}$ in arterial phase, 74.25 in venous phase, and $82 \mathrm{HU}$ in the delay phase. This illustrated a progressive enhancement pattern. In a study from Kim et al., (2004) they stated that Tubo Ovarian Abscess (TOA), one of which is caused by TB, caused enhancement of ovarian mass solid component.

Ascites was found in most patients $(81.9 \%)$. From all patients with ascites, $66.6 \%$ had free ascites and the rest had loculated ascites (33.3\%). This was similar to a study conducted by Uzunkoy et al.,(2004) which 
reported that all abdominal TB patients in their study had ascites and Wang et al., (2019) reported 73.33\% ascites prevalence. Ascites, one of clinical signs of abdominal tuberculosis, is characterized by fluid buildup in abdomen, swollen abdomen, and slightly raised tubercles of 1-2 $\mathrm{mm}$ across peritoneum. In Extra Pulmonary $\mathrm{TB}$, ascites progresses secondary to "exudation" of protein fluid from tubercles, similar to the mechanism that causes ascites in peritoneal carcinomatosis patients, and is often misdiagnosed in elderly patients. Most of peritonitis TB patients had ascites during diagnosis, while the rest showed an advanced, dry or fibroadhesive form of the disease (Uzunkoy et al., 2004).

Peritoneal thickening and enhancement were found in $68 \%$ of the patients. From all patients with peritoneal thickening and enhancement, most of them were smooth type (93.3\%). These were similar to a study by Wang et al., (2019) which reported peritoneal thickening in $40 \%$ of patients, a study by Demirkazik et al., (1996) which reported these findings in all patients, and a study by Sah et al., (2017) which reported these findings in $37.5 \%$ of patients. Peritoneal involvement is most often caused by infected lymph node rupture into peritoneal cavity, but it might also occur due to hematogenous and lymphatic spread. Another possible route is direct invasion of peritoneal lining from adjacent infected structures or caseus material release from fallopian tubes. Although literatures stated that pelvic mass, ascites, peritoneal thickening and enhancement, and omental involvement, could also lead to AOM and PID (Sah et al., 2017; Kim et al., 2004). Peritonitis TB is indicated by the presence of a smooth peritoneum with symmetrical thickening (Rodriguez and Pombo, 1996). Based on previous literatures, it could be considered that there is smooth peritoneal thickening and enhancement findings in abdominal TB compared to peritoneal carcinomatosis.

Omental involvement was found in $54 \%$ of patients. From all those patients, $58 \%$ had smudged involvement $25 \%$ had cake-like involvement, and $16.6 \%$ had nodular involvement. This was in accordance to a study from Shim et al., (2017) which reported omental thickening in 77.8\% abdominal TB patients. Omental involvement might occur in peritonitis TB. On CT-scan, omental involvement might present as omental nodules, infiltration diffusion, and omental thickening. Furthermore, it could also appear as an omental cake. Peritoneal carcinomatosis might also appear as oemental thickening. From a study by Charoensak et al., (2012) peritoneal carcinomatosis appeared as omental abnormality (71.7\%) with the most type being smudge type (36.8\%).

Lymphadenopathy was found only in $4.5 \%$ of patients. Lymph node enlargement in abdominal TB is usually associated with gastrointestinal TB and is rarely seen in peritoneal and solid organs. Lymph node disease pattern in abdominal TB is variable on CT scan, most of it often appears as lymph node enlargement (40-60\%) with hypoattenuation in the middle and hyperattenuation in the periphery (Rocha et al., 2015). However, another study by Bankier et al., (1995) reported adenopathy in only 1 out of 7 patients, therefore they concluded that low incidence of these findings suggested that the diagnostic value of this feature should not be too high. From all lymphadenopathy cases in this study, 62.5\% patients had central necrosis lymphadenopathy. This is in accordance to a literature which stated that central necrosis of lymph nodes is associated with caseation necrosis in TB case, but sometimes it occurs in metastatic lymphoma, in lymphoma, and in Whipple's disease (Rocha et al., 2015).

\section{Conclusion}

CT-scan findings in abdominal tuberculosis patients which resembled malignant ovarian tumors have similarities with findings in previous literatures, such as mass, ascites, and omental involvement findings. It is necessary to conduct a follow-up study with more samples to compare directly abdominal TB and malignant ovarian tumor CT findings, therefore each disease's characteristics could be determined.

\section{Acknowledgements}

I would like to dedicate my gratitude to all lecturers of Radiology, Faculty of Medicine Airlangga university, my family, and my friends for their endless support prayers 


\section{References}

Ahuja, V. (2019). Abdominal tuberculosis. UpToDate.

Anita Kumari, S. R., Pankaj, S., Choudhary, V., Anjili Kumari, S. R., Simi Kumari, S. R., Jaya Kumari, S. R., \& Syed Nazneen, S. R. (2017). Misdiagnosis of Ovarian Tuberculosis as Ovarian Carcinoma. Indian Journal of Gynecologic Oncology, 15(1), 8. https://doi.org/10.1007/s40944-017-0097-1

Bankier, A. A., Fleischmann, D., Wiesmayr, M. N., Putz, D., Kontrus, M., Hübsch, P., \& Herold, C. J. (1995). Update: Abdominal tuberculosis - Unusual findings on CT. Clinical Radiology, 50(4), 223-228. https://doi.org/10.1016/S0009-9260(05)83474-9

Charoensak, A., Nantavithya, P., \& Apisarnthanarak, P. (2012). Abdominal CT findings to distinguish between tuberculous peritonitis and peritoneal carcinomatosis. Journal of the Medical Association of Thailand, 95(11), 1449-1456.

Delgado, R., Fernandes, D., Ahouagi, A., \& Almeida, L. (2017). Peritoneal Tuberculosis Mimicking Advanced Ovarian Carcinoma with Peritoneal Carcinomatosis . Brazilian National Cancer Institute, Rio de Janeiro Single Center. 2.

Demirkazik, F. B., Akhan, O., Özmen, M. N., \& Akata, D. (1996). US and CT findings in the diagnosis of tuberculous peritonitis. Acta Radiologica, 37(4), 517-520. https://doi.org/10.3109/02841859609175435

KEMENKES, R. I. (2018). Infodatin: Pusat data dan informasi Kementrian Kesehatan RI): Jakarta. KEMENKES RI.

Kim, H. K., Kim, S. E., Park, M. I., Park, S. J., Moon, W., Kim, J. H., Jung, K., \& Nam, Y. J. (2017). A Case of Tuberculous Peritonitis Presenting as Small Bowel Obstruction. The Korean Journal of Gastroenterology = Taehan Sohwagi Hakhoe Chi, 69(5), 308-311. https://doi.org/10.4166/kjg.2017.69.5.308

Patel, S. M., Lahamge, K. K., Desai, A. D., \& Dave, K. S. (2012). Ovarian carcinoma or abdominal tuberculosis?-A diagnostic dilemma: Study of fifteen cases. Journal of Obstetrics and Gynecology of India, 62(2), 176-178. https://doi.org/10.1007/s13224-012-0163-7

Rocha, E. L. da, Pedrassa, B. C., Bormann, R. L., Kierszenbaum, M. L., Torres, L. R., \& D’Ippolito, G. (2015). Abdominal tuberculosis: a radiological review with emphasis on computed tomography and magnetic resonance imaging findings. Radiologia Brasileira, 48(3), 181-191. https://doi.org/10.1590/01003984.2013.1801

Rodriguez, E., \& Pombo, F. (1996). Peritoneal tuberculosis versus peritoneal carcinomatosis: distinction based on CT findings. Journal of Computer Assisted Tomography, 20(2), 269-272.

Ross, E., \& Fortin, C. (2016). Ovarian Cysts. The Cleveland Clinic Foundation.

Sah, S. K., Shi, X., Du, S., Li, X., Li, C. H., Shah, S., Shrestha, T. K., \& Li, Y. (2017). CT findings and analysis for misdiagnosis of female pelvic tuberculosis. Radiology of Infectious Diseases, 4(1), 19-25. https://doi.org/10.1016/j.jrid.2016.04.001

Shim, S. W., Shin, S. H., Kwon, W. J., Jeong, Y. K., \& Lee, J. H. (2017). CT differentiation of female peritoneal tuberculosis and peritoneal carcinomatosis from normal-sized ovarian cancer. Journal of Computer Assisted Tomography, 41(1), 32-38. https://doi.org/10.1097/RCT.0000000000000446

Thankam, R., \& Varma, M. D. (2008). Tuberculosis of the female genital tract. Glob Libr Women's Med.Kim, S. H., Kim, S. H., Yang, D. M., \& Kim, K. A. (2004). Unusual causes of tubo-ovarian abscess: CT and MR imaging findings. Radiographics, 24(6), 1575-1589. https://doi.org/10.1148/rg.246045016

Uzunkoy, A., Harma, M., \& Harma, M. (2004). Diagnosis of abdominal tuberculosis: Experience from 11 cases and review of the literature. World Journal of Gastroenterology, 10(24), 3647-3649. https://doi.org/10.3748/wjg.v10.i24.3647

Valentini, A. L., Gui, B., Miccò, M., Mingote, M. C., De Gaetano, A. M., Ninivaggi, V., \& Bonomo, L. (2012). Benign and suspicious ovarian masses-MR imaging criteria for characterization: Pictorial review. Journal of Oncology, 2012. https://doi.org/10.1155/2012/481806 
Wang, H., Qu, X., Liu, X., Ding, L., \& Yue, Y. (2019). Female peritoneal tuberculosis with ascites, pelvic mass, or elevated CA 125 mimicking advanced ovarian cancer: A retrospective study of 26 cases. Journal of the College of Physicians and Surgeons Pakistan, 29(6), 588-589. https://doi.org/10.29271/jcpsp.2019.06.588 WHO. (2014). Tuberculosis control in the South-East Asia region: annual TB report 2014. 